\title{
Quenched transmission of light through ultrathin metal films
}

\author{
Xiao, Sanshui; Mortensen, N. Asger
}

Published in:

Proceedings of SPIE - The International Society for Optical Engineering

Link to article, DOI:

$10.1117 / 12.893296$

Publication date:

2011

Document Version

Publisher's PDF, also known as Version of record

Link back to DTU Orbit

Citation (APA):

Xiao, S., \& Mortensen, N. A. (2011). Quenched transmission of light through ultrathin metal films. Proceedings of SPIE - The International Society for Optical Engineering, 8104, 81040B. https://doi.org/10.1117/12.893296

\section{General rights}

Copyright and moral rights for the publications made accessible in the public portal are retained by the authors and/or other copyright owners and it is a condition of accessing publications that users recognise and abide by the legal requirements associated with these rights.

- Users may download and print one copy of any publication from the public portal for the purpose of private study or research.

- You may not further distribute the material or use it for any profit-making activity or commercial gain

- You may freely distribute the URL identifying the publication in the public portal

If you believe that this document breaches copyright please contact us providing details, and we will remove access to the work immediately and investigate your claim. 


\title{
Quenched transmission of light through ultrathin metal films
}

\author{
Sanshui Xiao and N. Asger Mortensen \\ DTU Fotonik, Department of Photonics Engineering, \\ Technical University of Denmark, DK-2800 Kongens Lyngby, Denmark.
}

\begin{abstract}
We discuss optical properties of ultrathin metal films, with particular attention to the phenomenon of quenched transmission. Transmission of light through an optically ultrathin metal film with a thickness comparable to its skin depth is significant. We demonstrate the quenched transmission through the ultrathin metal films when they are periodically modulated. We also discuss the physics behind it and explain how this abnormal phenomenon is ascribed to surface plasmon resonance effects.
\end{abstract}

Keywords: diffraction and gratings, optics at surfaces

\section{INTRODUCTION}

Transmission of light through an otherwise optically opaque metal film is quite weak. By the presence of periodic subwavelength holes, the opaque metal film (i.e. thickness larger than $100 \mathrm{~nm}$ ) can pass extraordinary amounts of light at certain frequencies, so-called extraordinary optical transmission, which was first reported by Ebbesen et al. ${ }^{1}$ The mechanisms behind it have been widely addressed and it has been shown that the enhanced transmission is mostly attributed to the surface plasmon polaritons (SPPs). ${ }^{2-7}$ Transmission of light is prominent for an ultrathin metal film with the thickness comparable to its optical penetration depth. When periodically modulated by subwavelength apertures, intuitively one expects that the ultrathin film could transmit even more light since less material is blocking the light. To much surprise, it was recently predicted by means of analytical and numerical calculations ${ }^{8}$ that the transmission can nevertheless be totally suppressed. This nontrivial phenomenon is opposite to the extraordinary optical transmission of light. Subsequently, the suppressed transmission of light through ultrathin films pierced with periodic arrays of subwavelength apertures was experimentally demonstrated. ${ }^{9}$ Numerical calculations ${ }^{10}$ also pointed out that nearly-zero transmission can be realized by a two-dimensional array of isolated metal disks, while an ultrathin metal film modulated by a two-dimensional subwavelength hole array does not support a nearly zero transmission band. In this paper, we experimentally demonstrate nearly-null transmission of light through ultrathin metal film when periodically modulated. Experimental results are verified by numerical simulations. The suppressed transmission is associated with the resonance anomaly (one of Wood's anomalies ${ }^{11-13}$ ), which is attributed to the excitation of the surface plasmon polariton (SPP) resonance.

\section{FABRICATION}

Figure 1 illustrates the ultrathin metal films modulated by one-dimensional slits and a two-dimensional disk array. The samples were prepared with a standard electron beam lithography (EBL) technique, as illustrated in Fig.2 (a). We started with a $1 \mathrm{~mm}$ quartz wafter. First, a $100 \mathrm{~nm}$ ZEP520A (3.6\%, Zeon Corp., Tokyo, Japan) resist was spincoated onto a $1 \mathrm{~mm}$ quartz substrate. Then, a $15 \mathrm{~nm}$ aluminium layer was thermally deposited on top of the ZEP layer to prevent charge accumalation during the following EBL step. The EBL exposure was done with a $100 \mathrm{kV}$ JEOL JBX-9300FS EBL tool $\left(200 \mu \mathrm{C} / \mathrm{cm}^{2}\right.$ dose, $2 \mathrm{nA}$ current, $6 \mathrm{~nm}$ spotsize). The aluminium layer was removed in undiluted MF-322 (Rohm and Haas, Coventry, UK) and the positive ZEP resist was developed in ZED-N50 (Zeon Corp.) developer. Then a brief descum process was carried out to remove any residual resist. Finally, $5 \mathrm{~nm}$ titanium and $15 \mathrm{~nm}$ gold were deposited before lift-off was performed concluding the fabrication process. The scanning electron microscopy images of the grating structure and gold disk array are shown in Fig. 2 (b) and Fig. 2 (c), respectively. Fig. 2 (b) illustrates the one-dimensional periodic array of

Further author information: Send correspondence to saxi@fotonik.dtu.dk or asger@mailaps.org

Nanostructured Thin Films IV, edited by Raúl J. Martín-Palma, Yi-Jun Jen, Tom G. Mackay,

Proc. of SPIE Vol. 8104, 81040B · C) 2011 SPIE · CCC code: 0277-786X/11/\$18 · doi: 10.1117/12.893296 

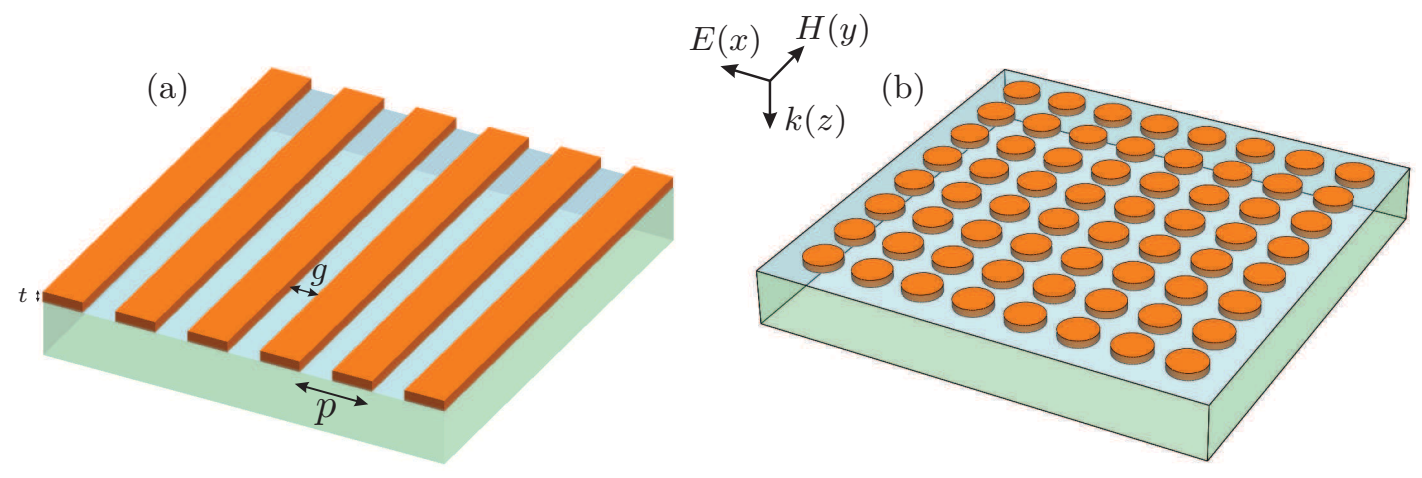

Figure 1. A schematic illustration of (a) a quartz wafer with an ultrathin metal film perforated by a one-dimensional periodic array of subwavelength slits; (b) an ultrathin circular disk array deposited on a quartz wafter. Axis show the propagation direction and polarization of the incident plane wave.

(a)

(1)

(2)

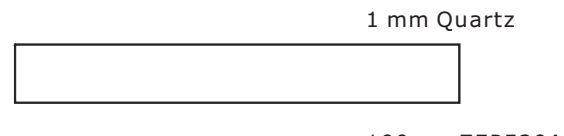

(3)

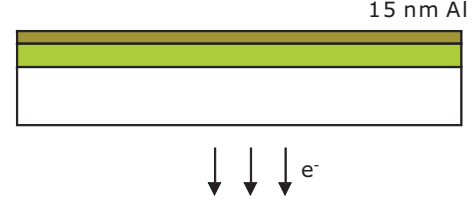

(4)

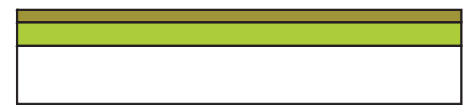

(b)

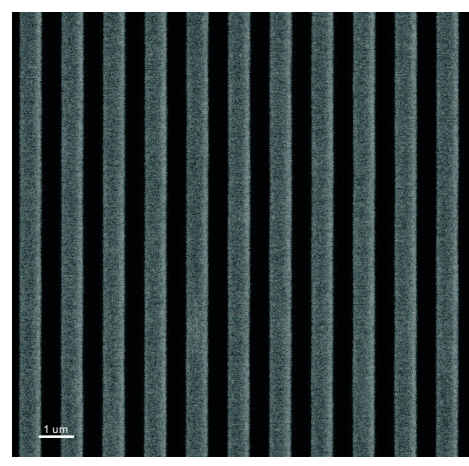

Undiluted MF322

(5)

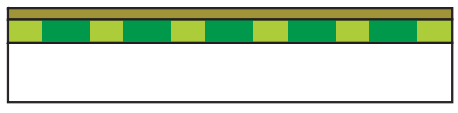

NED-N50

(6)

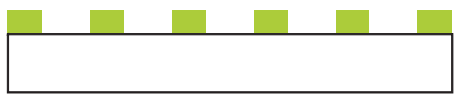

(7)

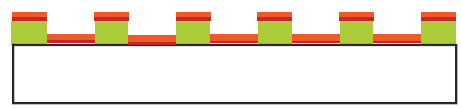

Lift-off

(8)

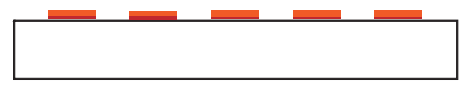

(c)

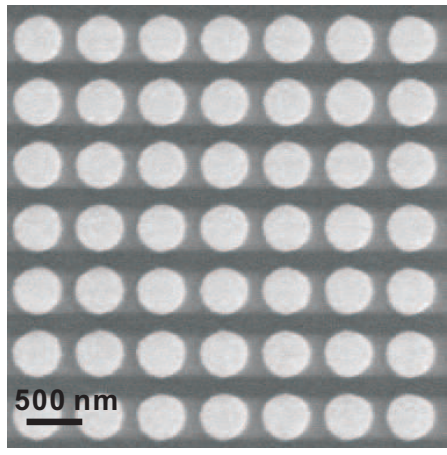

Figure 2. (a) Schematic drawing of the processflow. (1) Quartz wafer. (2) Spincoat of ZEP520A. (3) Deposition of Al. (4) EBL exposure. (5) Removal of Al. (6) Development. (7) Metal deposition. (8) Lift-off. (b) Scanning electron microscope image of the grating structure; (c) Scanning electron microscope image of the gold disk array. 

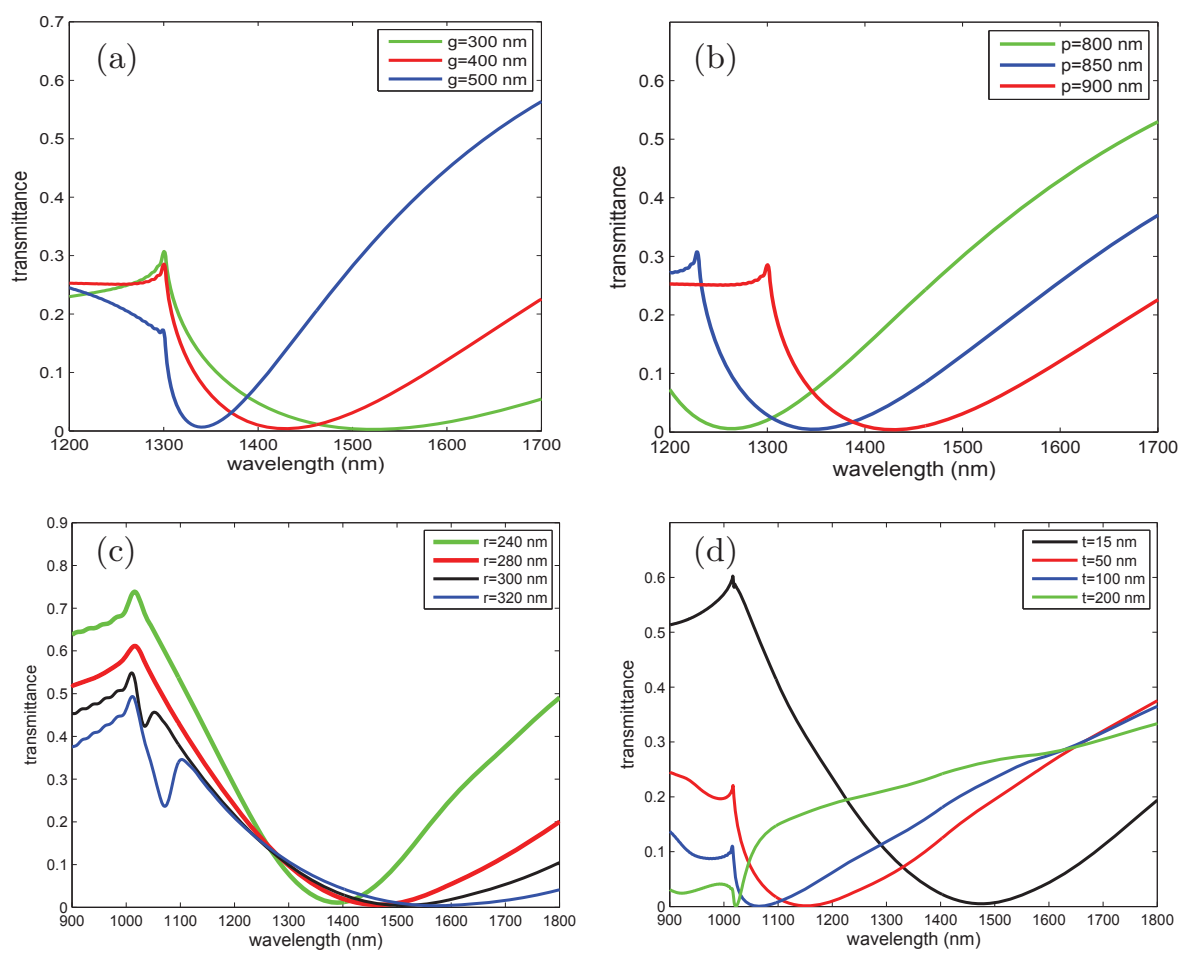

Figure 3. (a) Simulated transmittance as a function of the gap $g$ for the grating structure when $p=900 \mathrm{~nm}$. (b) Simulated transmittance as a function of the periodicity $p$ of the grating structure when $g=400 \mathrm{~nm}$. (c) Simulated transmittance as a function of the period $p$ for the disk array. (d) Simulated transmittance spectra as a function of the thickness $t$ for the disk array.

subwavelength slits perforated in an ultrathin metal film on the quartz wafter, where the thickness of the film is $t=15 \mathrm{~nm}$, the width of the slits is $g=400 \mathrm{~nm}$, and the period of the slit array is $p=900 \mathrm{~nm}$. Fig. 2 (c) illustrates gold disk array deposited on the quartz wafter, where the thickness of the film is $15 \mathrm{~nm}$, the radius of the disk $r$ is $r=262.5 \mathrm{~nm}$, and the period of the array is $p=700 \mathrm{~nm}$.

\section{NUMERICAL SIMULATIONS}

Numerical simulations were performed with the aid of a commercially available CST MicroStudio package. ${ }^{14}$ For the optical properties of gold, we used the experimental data from Ref. ${ }^{15}$ The refractive index of the quartz was chosen as 1.445. Due to the periodicity and symmetry conditions in the $x-y$ plane, we used the perfect electric conductor and perfect magnetic conductor in the $x-y$ plane, equivalent to the periodic boundary condition for the normal incidence, and applied perfect matched layers as the boundaries conditions in the propagation direction. Figure 3 (a) and 3 (b) show the transmittance when varying the size of the slits $g$ and the period of the structure $p$ for the one-dimensional grating, as shown in Fig.1 (a). The resonant wavelength associated with the suppressed optical transmission is blueshifted with increasing $g$. When enlarging $p$, the resonance is redshifted. For the structured film at the resonance $\lambda=1428.6 \mathrm{~nm}$, the transmittance $(T)$, reflectance $(R)$, and absorption $(A)$ are $0.39 \%, 89.8 \%$, and $9.81 \%$, respectively. Note that $T=10.21 \%, R=85.16 \%$, and $A=4.63 \%$ for the case of the closed film. We conclude that the excitation of the SPPs results in the suppression of the transmittance and increase of the reflectance as well as the absorption. We can also observe from Figs. 3 (a) and 3 (b) that the Rayleigh anomaly (RA) is independent on $g$, while strongly dependent on $p$. The wavelengths associated with the Rayleigh anomaly are $1156 \mathrm{~nm}, 1228 \mathrm{~nm}$, and $1300 \mathrm{~nm}$ for $p=800 \mathrm{~nm}, p=850 \mathrm{~nm}$, and $p=900 \mathrm{~nm}$, respectively. The results are in accordance with expectations, since the wavelength values with the Rayleigh anomaly are equal to the product of the grating period and the refractive index of the substrate. 


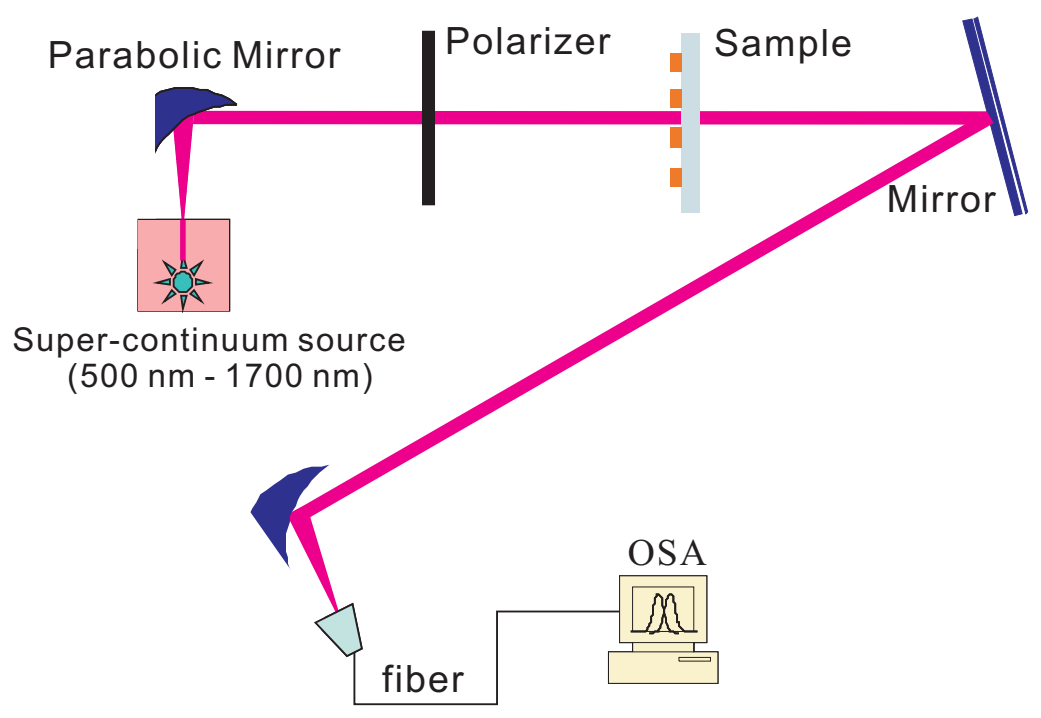

Figure 4. Free space measurement setup

For the case of the gold disk array, the transmittance as a function of the period is shown in Fig. 3 (c). The transmittances are also nearly zero at the resonant positions and the RAs coincide with the predicted spectral position. Figure 3 (d) illustrates how the resonances shift when tuning the thickness of the gold disk. The resonances are blue-shifted when increasing the thickness. As mentioned above, the SPP modes guided by the two interfaces strongly couple, while they are almost decoupled for a thick film, i.e., $t=200 \mathrm{~nm}$. Under this circumstance, the SPP resonance is only related to the one for a single interface. From the dispersion of the SPP at an interface between a dielectric and a metal, one knows that the SPP resonance is very close to the RA as shown by the dashed line in Fig. 3 (d). Meanwhile, the transmittances for all cases are nearly zero at the resonances. Besides, it can be seen in Fig. 3 (d) that the RAs are independent of the film thickness.

\section{EXPERIMENTAL CHARACTERIZATION}

The fabricated samples were characterized in a free space measurement setup using a super-continuum light source (500-1750 nm, SuperK SCB-Compact 100-PC), as illustrated in Fig. 4. First, the emitted beam from the light source was collimated and broadened via a parabolic mirror. Then, the light was linearly polarized by a polarizer before reaching the sample. The transmitted light was reflected by a mirror and focused by another parabolic mirror and finally collected by a fiber leading to a spectrum analyzer (400-1750 nm, Ando AQ-6315E). Note that under this circumstance only the zero-order transmittance was measured.

\section{RESULTS AND DISCUSSION}

The red lines in Fig. 5 (a) and Fig. 5 (b) represent the experimental results of the transmittance spectra for the closed and modulated ultrathin metal films at normal incidence, respectively. At the wavelength of our interest as shown in Figs. 5 (a) and 5(b), one can observe that around $10 \%$ of the intensity is transmitted through the closed metal film with the thickness of $15 \mathrm{~nm}$, as shown in Fig. 5 (a). When periodically modulated by an array of one-dimensional slits, transmission is dramatically suppressed. Especially for the wavelength around $1428.6 \mathrm{~nm}$, the transmittance decreases significantly from $6.7 \%$ to $2.1 \%$. Intuitively one expects that the metal film with slits, compared with the closed film, would transmit more light, since less material is blocking the light. However, we emphasize that the measurement demonstrates the opposite: less light is transmitted through the perforated film! This nontrivial effect is also verified by numerical simulations, shown by the blue lines in Figs. 5 (a) and $5(\mathrm{~b})$.

Fig. 5 (c) shows the measured transmittances at normal incidence for different sizes of the gold disk arrays. As represented in Fig. 5 (c), one can observe that the transmission is significantly suppressed, especially for the case 

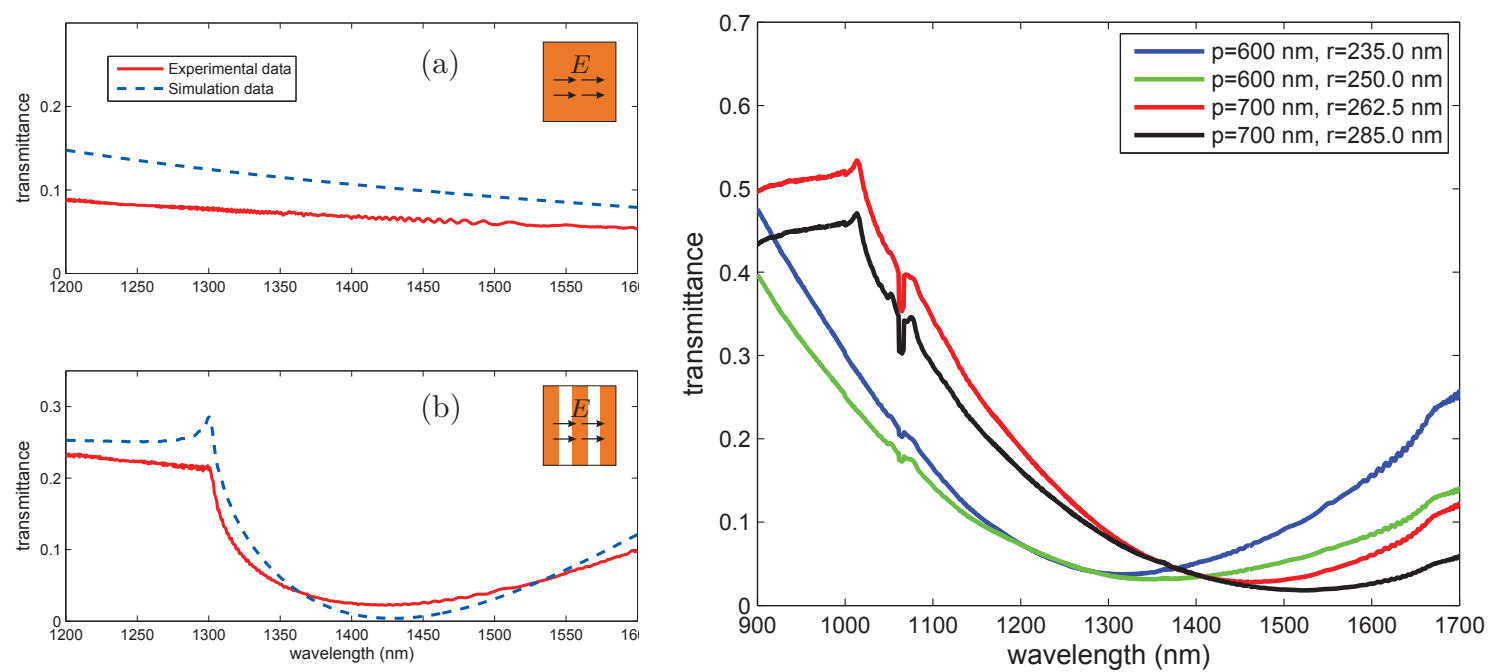

Figure 5. Transmittance spectra for the closed (a) and one-dimensional perforated (b) ultrathin gold film at normal incidence. The red lines in (a) and (b) correspond to the experimental results and the blue lines are the results from numerical analysis. Measured transmittance spectra (c) for periodic arrays of metal disks when tuning the structure parameters.

with $p=700 \mathrm{~nm}$ and $r=285.0 \mathrm{~nm}$. The transmittance is almost totally suppressed at the resonant wavelength $\lambda=1524 \mathrm{~nm}$. Compared to the case of the unstructured metal film with the same thickness, the transmittance drops from $6.7 \%$ to $1.52 \%$. The result shows an opposite phenomenon as compared with the extraordinary optical transmission of the light through the film when perforated by the subwavelength holes..$^{3,5-7,16-18}$ Intuition might suggest that the metal disk array, compared with the closed film, would transmit more light. However, we emphasize that the measurement demonstrates less light transmitted through the gold disks array. When varying $p$ or $r$, the resonant wavelength slightly changes, as well as the transmittance. The resonant wavelength related to the suppressed transmission is both redshifted when increasing $p$ and $r$.

Similar to the result for the reflectance of the surface-corrugated metal film, ${ }^{11-13,19}$ the strongly suppressed transmission is related to the resonance anomaly (one of Wood's anomalies), which is due to the excitation of the SPP resonance along the surface. The periodic structure of the gold disk array enables light to couple to SPPs via reciprocal wave vectors. ${ }^{20}$ The SPP resonance appears when the wavevector of the surface plasmon matches the wave vector of the incident photon and grating as $k_{\mathrm{sp}}=k_{\|} \pm n G_{x} \pm m G_{y}$, where $k_{\mathrm{sp}}$ is the surface plasmon wavevector, $k_{\|}$is the component of the incident wavevector in the plane of the grating, and $G_{x}=G_{y}=2 \pi / p$ is the grating reciprocal vector for the square array. For an ultrathin film with a thickness $t$ of $15 \mathrm{~nm}$, the SPP modes guided by the two interfaces strongly hybridize due to the overlap of their fields inside the metal. From Maxwell's equations and boundary conditions, the dispersion relation for the ultrathin film can be derived for these coupled modes: ${ }^{21}$

$$
\tanh \left(\alpha_{m} t\right)=-\frac{\varepsilon_{\mathrm{m}} \alpha_{m}\left(\varepsilon_{\mathrm{d} 1} \alpha_{\mathrm{d} 2}+\varepsilon_{\mathrm{d} 2} \alpha_{\mathrm{d} 1}\right)}{\varepsilon_{\mathrm{d} 1} \varepsilon_{\mathrm{d} 2} \alpha_{\mathrm{m}}^{2}+\varepsilon_{\mathrm{m}}^{2} \alpha_{\mathrm{d} 1} \alpha_{\mathrm{d} 2}} .
$$

Here, $\alpha_{\mathrm{d} 1}^{2}=k_{\mathrm{sp}}^{2}-\varepsilon_{\mathrm{d} 1} k_{0}^{2}, \alpha_{m}^{2}=k_{\mathrm{sp}}^{2}-\varepsilon_{\mathrm{m}} k_{0}^{2}$, and $\alpha_{\mathrm{d} 2}^{2}=k_{\mathrm{sp}}^{2}-\varepsilon_{\mathrm{d} 2} k_{0}^{2}$ with $k_{0}$ being the the incident photon wave number in vacuum. For the structures we investigate here, $\varepsilon_{\mathrm{d} 1}=1$ (air) and $\varepsilon_{\mathrm{d} 2}=2.088$ (fused glass) and therefore $\varepsilon_{\mathrm{d} 1}<\varepsilon_{\mathrm{d} 2}$. For this strongly asymmetric geometry, the ultra-thin metallic film only supports a single strongly damped short range surface plasmon mode. For example, when $\lambda=1524 \mathrm{~nm}$, the effective refractive index of the SPP mode is around $1.50-0.011 j$. Of course, for an in-plane modulated thin film, Eq. (1) will be subject to band-folding phenomena associated with the finite value of the reciprocal lattice vector $G$. 


\section{SUMMARY}

We have experimentally demonstrated nearly-zero transmission of light through the ultrathin metal film, which is periodically modulated by a one-dimensional periodic array of subwavelength slits and the gold disk arrays. The experimental results have been verified by numerical simulations, and the suppressed transmission is attributed to the excitation of surface plasmon-polariton waves. We have also shown the Rayleigh anomalies, having the same positions as those predicted theoretically. The suppressed transmission results in the enhanced absorption, which is potentially interesting in the context of energy harvesting for solar cells and photocatalysis.

\section{ACKNOWLEDGMENTS}

This work is partly supported by the Danish Research Council for Technology and Production Sciences (grant no: 274-07-0379) and the Catalysis for Sustainable Energy initiative Center, funded by the Danish Ministry of

Science, Technology and Innovation. S. Xiao would like to acknowledge the travel grant from Otto Mønsteds Fond.

\section{REFERENCES}

1. Ebbesen, T. W., Lezec, H. J., Ghaemi, H. F., Thio, T., and Wolff, P. A., "Extraordinary optical transmission through sub-wavelength hole arrays," Nature 391, 667-669 (1998).

2. Ghaemi, H. F., Thio, T., Grupp, D. E., Ebbesen, T. W., and Lezec, H. J., "Surface plasmons enhance optical transmission through subwavelength holes," Phys. Rev. B 58, 6779-6782 (1998).

3. Porto, J. A., Garcia-Vidal, F. J., and Pendry, P. B., "Transmission resonances on metallic gratings with very narrow slits," Phys. Rev. Lett. 83, 2845-2853 (1999).

4. Martin-Moreno, L., Garca-Vidal, F. J., Lezec, H. J., Pellerin, K. M., Thio, T., Pendry, J. B., and Ebbesen, T. W., "Theory of extraordinary optical transmission through subwavelength hole arrays," Phy. Rev. Lett. 86, 1114-1117 (2001).

5. Xiao, S. and Qiu, M., "Theoretical study of the transmission properties of a metallic film with surface corrugations," J. Opt. A: Pure Appl. Opt. 9, 348-351 (2007).

6. García de Abajo, F. J. and Sáenz, J. J., "Electromagnetic surface modes in structured perfect-conductor surfaces," Phys. Rev. Lett. 95, 233901 (2005).

7. Xiao, S., Peng, L., and Mortensen, N. A., "Enhanced transmission of transverse electric waves through periodic arrays of structured subwavelength apertures," Opt. Express 18, 6040-6047 (2010).

8. Spevak, I. S., Nikitin, A. Y., Bezuglyi, E. V., Levchenko, A., and Kats, A. V., "Resonantly suppressed transmission and anomalously enhanced light absorption in periodically modulated ultrathin metal films," Phys. Rev. B 79, 161406 (2009).

9. Braun, J., Gompf, B., Kobiela, G., and Dressel, M., "How holes can obscure the view: Suppressed transmission through an ultrathin metal film by a subwavelength hole array," Phy. Rev. Lett. 103, 203901 (2009).

10. Sun, Z., Zuo, X., and Lin, Q., "Plasmon-induced nearly null transmssion of light through gratings in very thin metal films," Plasmonics 5, 13-19 (2010).

11. Wood, R. W., "On a remarkable case of uneven distribution of light in a diffraction grating spectrum," Phil. Mag. 4, 396-402 (1902).

12. Fano, U., "The theory of anomalous diffraction gratings and of quasi-stationary waves on metallic surfaces (sommerfeld's waves)," J. Opt. Soc. A 31, 213-222 (1941).

13. Hessle, A. and Oliner, A. A., "A new theory of wood's anomalies on optical gratings," App. Opt. 4, 12751297 (1965).

14. CST MICROWAVE STUDIO, CST GmbH, Germany .

15. Johnson, P. B. and Christy, R. W., "Optical constants of the nobal metals," Phys. Rev. B 6(12), 4370-4379 (1972).

16. Xiao, S., Mortensen, N. A., and Qiu, M., "Enhanced transmission through arrays of subwavelength holes in gold films coated by a finite dielectric layer," J. Eur. Opt. Soc., Rapid Publ. 3, 08022 (2008). 
17. Xiao, S., Zhang, J., Peng, L., Jeppesen, C., Malureanu, R., Kristensen, A., and Mortensen, N. A., "Nealyzero transmission through periodically modulated ultrathin metal films," Appl. Phys. Lett. 97, 071116 (2010).

18. Xiao, S. and Mortensen, N. A., "Surface-plasmon-polariton-induced suppressed transmission through ultrathin metal disk arrays," Opt. Lett. 36, 37-39 (2011).

19. Rayleigh, L. O. M., "Note on the remarkable case of diffraction spectra described by prof. wood," Phil. Mag. 6(14), 60-65 (1907).

20. Raether, H., [Surface plasmons on smooth and rough surfaces and on gratings], Springer, Berlin (1988).

21. Yang, F., Sambles, J. R., and Bradberry, G. W., "Long-range surface modes supported by thin films," Phys. Rev. B 44, 5855-5872 (1991). 